\title{
Short-Term Outcomes for the Biologic Treatment of Bone Marrow Edema of the Knee Using Bone Marrow Aspirate Concentrate and Injectable Demineralized Bone Matrix
}

\author{
Connor S. Kasik, D.O., Stephen Martinkovich, M.D., Brian Mosier, M.D., and \\ Sam Akhavan, M.D.
}

Purpose: To evaluate short-term outcomes for the biologic treatment of bone marrow edema (BME) of the knee using bone marrow aspirate concentrate (BMAC) and injectable demineralized bone matrix (iDBM). Methods: We performed a review of prospectively collected data from patients who underwent treatment for bone marrow lesions (BMLs) of the knee using BMAC and iDBM (IntraOsseous BioPlasty; Arthrex, Naples, FL) between May 2017 and December 2018. Inclusion criteria included patients aged 18 to 65 years with the presence of BME on T2-weighted magnetic resonance imaging in the subchondral weightbearing region of the tibia or femoral condyle, with pain corresponding to the same compartment. The International Knee Documentation Committee (IKDC), pain visual analog scale (VAS), and 12-Item Short Form Health Survey (SF-12) scores were used to evaluate clinical outcomes. Results: We evaluated 20 patients who were treated at a single academic medical institution over a mean 14.5-month follow-up (median, 14 months; range, 6-25 months). The average patient age was 51.7 years (range 38-62 years). Compared with preoperative values, the visual analog scale decreased from 7.0 to $1.3(P=$ .008). The mean International Knee Documentation Committee scores improved from 29.2 to $66.1(P=.063)$. Both the Physical and Mental Component Scores of the 12-Item Short Form Health Survey also showed improvement (Physical Component Score, $P=.438$; Mental Component Score, $P=.563$ ). Based on postoperative magnetic resonance imaging, $75 \%$ ( 3 of 4 ) of the BMLs demonstrated complete healing. The survival rate was $93 \%$ at l-year follow-up. Conclusion: Biologic treatment of BME of the knee using BMAC and iDBM is an effective adjunct to arthroscopy that provides short-term pain relief for BMLs associated with degenerative conditions of the knee. This procedure is associated with clinically significant improvements in knee pain and function at a short-term follow-up. Level of Evidence: Level IV, therapeutic case series.

\section{Introduction}

B one marrow lesions (BMLs), also known as bone marrow edema (BME), are defects of subchondral bone that can occur as a result of acute or chronic

From the Department of Orthopaedics, Allegheny General Hospital, Pittsburgh, Pennsylvania, U.S.A. (C.S.K., S.M., S.A.); and Department of Orthopaedics, Allegheny Health Network Forbes Hospital, Monroeville, Pennsylvania, U.S.A. (B.M.)

The authors report the following potential conflicts of interest or sources of funding: S.A. has spoken on this research topic for the speaker's bureau of Arthrex and served as a consultant for Arthrex. Full ICMJE author disclosure forms are available for this article online, as supplementary material.

Received May 19, 2019; accepted July 1, 2019.

Address correspondence to Connor S. Kasik, D.O., 1307 Federal St., Floor 2, Pittsburgh,PA 15212,U.S.A.E-mail:connorkasik@gmail.com

(C) 2019 by the Arthroscopy Association of North America. Published by Elsevier Inc. This is an open access article under the CC BY-NC-ND license

(http://creativecommons.org/licenses/by-nc-nd/4.0/).

2666-061X/19618

https://doi.org/10.1016/j.asmr.2019.07.001 traumatic injury, subchondral insufficiency fractures of the knee (SIFK), stress injuries, osteoarthritis, or osteonecrosis. BMLs are found on magnetic resonance imaging (MRI), characterized by increased signal on T2-weighted fat suppression sequences (Fig 1). ${ }^{1,2}$ The presence of BME is typically the result of mechanically and/or histologically altered subchondral bone. The physiologic remodeling of subchondral bone fails as a result of ongoing joint forces, increased focalization of stress, and reduced healing capacity of the subchondral bone. ${ }^{1,3-6}$ Histologic evaluations of these lesions demonstrate changes consistent with a chronic stress fracture, including fibrosis, medullary fat necrosis, trabecular bone microfractures, and poor mineralization. ${ }^{5}$ These changes in subchondral bone are currently recognized as an important factor in the process of osteoarthritis. ${ }^{7,8} \mathrm{BML}$ development is associated with subchondral bone attrition and loss of mechanical integrity, leading to progression of cartilage loss and rapid joint deterioration. ${ }^{9-11}$ 
The etiology and pathogenesis of BMLs of the knee are important to understand to drive treatment. Historically, spontaneous osteonecrosis of the knee (SONK) has been thought to occur secondary to ischemia, resulting in necrosis. However, recent evidence has demonstrated that it may be caused by subchondral insufficiency fractures in osteopenic bone. ${ }^{12} \mathrm{~A}$ subchondral insufficiency fracture of the knee (SIFK) is a subchondral fracture in the weightbearing area of the knee caused by haversion and trabecular collapse. ${ }^{13}$ These insufficiency fractures lead to fluid accumulation in the bone marrow, which results in subsequent edema and then focal ischemia and eventual necrosis. ${ }^{12}$ Factors predisposing to insufficiency fractures may include meniscal tears or meniscectomy, which can alter the native biomechanics and increase contact pressures and stress concentration on weightbearing areas. $^{12,13}$

$\mathrm{BME}$ is a known negative prognostic factor that is strongly associated with pain, decreased function, cartilage damage, structural deterioration, accelerated osteoarthritis, and rapid progression to total knee arthroplasty (TKA). ${ }^{6,9,14-17}$ Therefore, there is an interest in therapies directed toward the treatment of subchondral bone that aims to preserve the joint.

Percutaneous treatment of BME of the knee using BMAC and $\mathrm{IDBM}$ is a minimally invasive, fluoroscopically assisted procedure that directly targets BMLs from subchondral bone defects with techniques that encourage physiologic bone remodeling. This procedure borrows from the treatment principle that has been previously studied in the treatment of avascular necrosis of the hip. ${ }^{18,19}$ The technique involves the direct injection of a biologic mixture of BMAC and IDBM as augmentation to core decompression of a subchondral bone defect of the knee. This orthobiologic mixture acts to repair and restore normal bone anatomy and function. These factors may be particularly important for younger, active patients who wish to reduce pain, retain function, and avoid joint replacement surgery.

The purpose of this study was to evaluate short-term outcomes for the biologic treatment of BME of the knee using BMAC and iDBM. We hypothesized that this procedure will result in decreased pain and an improvement in knee patient-reported functional outcomes.

\section{Methods}

\section{Patient Selection}

The study was approved by our institutional review board (IRB 2018-249) to review prospectively collected data and follow-up information, and informed consent was obtained from all patients at the time they were entered in the database.

Between May 2017 and December 2018, all patients who were treated for symptomatic BMLs of the knee by
2 sports medicine fellowship-trained attending orthopaedic surgeons at a single academic medical institution were included in the study. Inclusion criteria included patients aged 18 to 65 years old with the presence of BME on T2-weighted MRI in the subchondral weightbearing region of the tibia or femoral condyle, with pain corresponding to the same compartment as the BML. Exclusion criteria included autoimmune diseases, mechanical lower extremity malalignment $>8$ degrees, and radiographic evidence of Kellgren-Lawrence grade 4 osteoarthritis.

Patients were evaluated through physical examination, radiography, and MRI. After clinical evaluation, the appropriate patients were informed about the benefits and limitations of the aforementioned procedure. Surgical indications included the presence of BMLs observed on MRI involving weightbearing regions of the knee with associated localized pain on weightbearing and palpation. Patients also had to fail 3 months of conservative therapy, including oral nonsteroidal anti-inflammatory drugs, physical therapy, bracing, and injectable therapies consisting of intra-articular corticosteroids and viscosupplementation. Working preoperative diagnoses of patients included SIFK and SONK.

\section{Surgical Technique}

This technique is performed with the patient in the supine position. The ipsilateral hip is sterilely prepped and draped in anticipation for the iliac crest bone marrow aspiration. A Jamshidi needle is inserted percutaneously into the anterior iliac crest. Two large syringes are subsequently filled until a total of $60 \mathrm{~mL}$ of bone marrow is obtained. This bone marrow aspirate is subsequently placed into the bone marrow processing system (Angel System, Arthrex, Naples, FL) to obtain 4 to $5 \mathrm{~mL}$ of BMAC.

An initial diagnostic knee arthroscopy is performed through standard anteromedial and anterolateral portals, and all corresponding intra-articular pathology is addressed at this time. Patients with concomitant medial or lateral meniscal tears are treated with partial meniscectomies. Cartilage defects are treated with either chondroplasty or other appropriate marrowstimulating procedures (e.g., microfracture or abrasion chondroplasty) depending on the size, grade, or location of the lesion. Osteochondral defects grade III or IV that are $\leq 2 \mathrm{~cm}^{2}$ are treated with microfracture. Large uncontained grade III and IV chondral lesions that are $>2 \mathrm{~cm}^{2}$ are treated with abrasion arthroplasty. Loose joint bodies are removed arthroscopically. Chondroplasty is used when incidental findings reveal irregular loose chondral flaps that are $<1$ to $2 \mathrm{~cm}^{2}$. After arthroscopy, a $4.5-\mathrm{mm}$ cannulated drill is inserted percutaneously into the knee BML by using intraoperative fluoroscopy corresponding to the location as mapped with the use of MRI and preoperative examination correlating with the patient's bony pain (Fig 2). 
Fig 1. Bone medullary lesion involving the medial tibial plateau of the right knee with hypersignal on coronal (A) and sagittal (B) T2-weighted magnetic resonance imaging, consistent with subchondral insufficiency fracture of the knee.
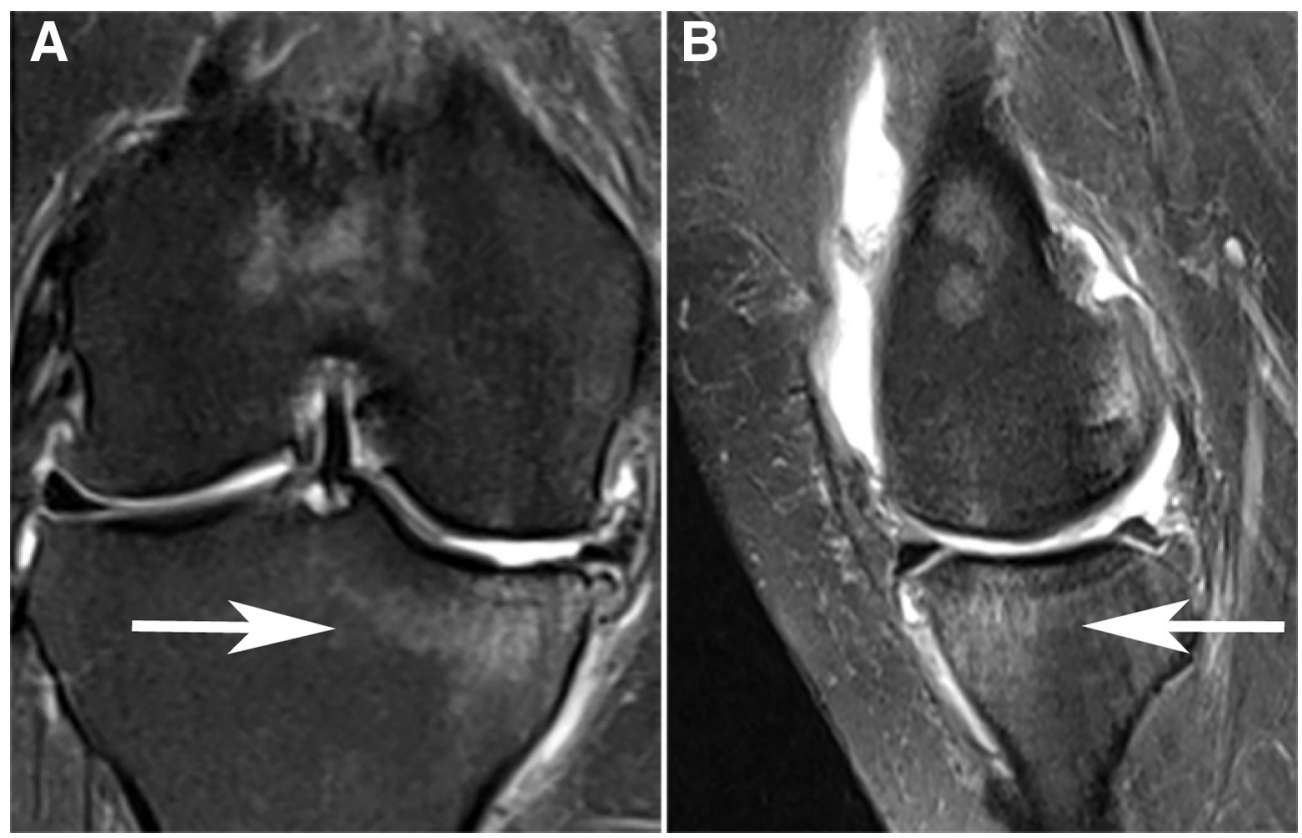

The authors usually insert the percutaneous drill on the same side as the lesion, to promote ease of the procedure when obtaining orthogonal imaging. However, femoral lesions may be approached from the opposite side. The cannulated drill is then advanced to the center of the BML. Care is taken to remain subchondral in nature and to advance deep enough so that the fenestrations of the side delivery system of the cannulated drill are fully contained by bone. This will decrease the chance of fluid extravasation into the soft tissues. The location of the drill is confirmed on both anteroposterior and lateral fluoroscopic imaging. The central drill guidepin is subsequently removed to complete the core decompression.

Next, $3 \mathrm{~mL}$ of BMAC is mixed with $5 \mathrm{~mL}$ of iDBM (Allosync PURE; Arthrex) using a mixing and delivery syringe. After thorough mixing, this mixture is transferred to $1-\mathrm{mL}$ syringes and injected through the cannulated drill and delivery system. The inner stylus is inserted through the delivery cannula to deliver the last $1 \mathrm{~mL}$ of mixture into the BML. For patients with bipolar lesions, a second cannulated drill is then inserted percutaneously corresponding to the location of the second BML. The process involving the injection of the biologic mixture is then repeated. A repeat diagnostic arthroscopy is then performed to verify that the biologic mixture was inserted into bone and did not extravasate into the knee joint.

\section{Postoperative Rehabilitation Protocol}

Postoperatively, patients are discharged on the same day. In cases of isolated treatment of BME of the knee using solely BMAC and iDBM, patients are placed into a hinged knee brace locked in extension and instructed to remain non-weightbearing for 3 weeks. Weightbearing is then advanced to full and the brace is discontinued. Formal physical therapy is initiated and typically lasts for 4 to 8 weeks. Patients are permitted to return to most activities of daily living once they are full weightbearing, typically by 4 to 6 weeks. In cases of concomitant procedures performed, the rehabilitation process for the biologic treatment using BMAC and iDBM takes precedence.

\section{Clinical Outcome Evaluation}

Patients were evaluated with a range of functional scores, including the visual analog scale (VAS) for pain, International Knee Documentation Committee (IKDC), and 12-Item Short Form Health Survey (SF-12). Patients also answered questions regarding self-reported knee function. Scores were gathered preoperatively and at 2 weeks, 6 weeks, 3 months, 6 months, and 1 year postoperatively during consultations or phone interview or via a mailed questionnaire. Patients were excluded if they were not available for follow-up visits, phone interview, or questionnaire. Furthermore, patients who did not fully complete the patient-reported outcome measurements (PROMs) were excluded. Patients who complained of knee pain were brought in for a follow-up clinical evaluation. A postoperative MRI was also obtained at 6 months postoperatively in patients willing to undergo MRI. The interval status of the BML on the postoperative MRI was categorized as healed or not healed. 

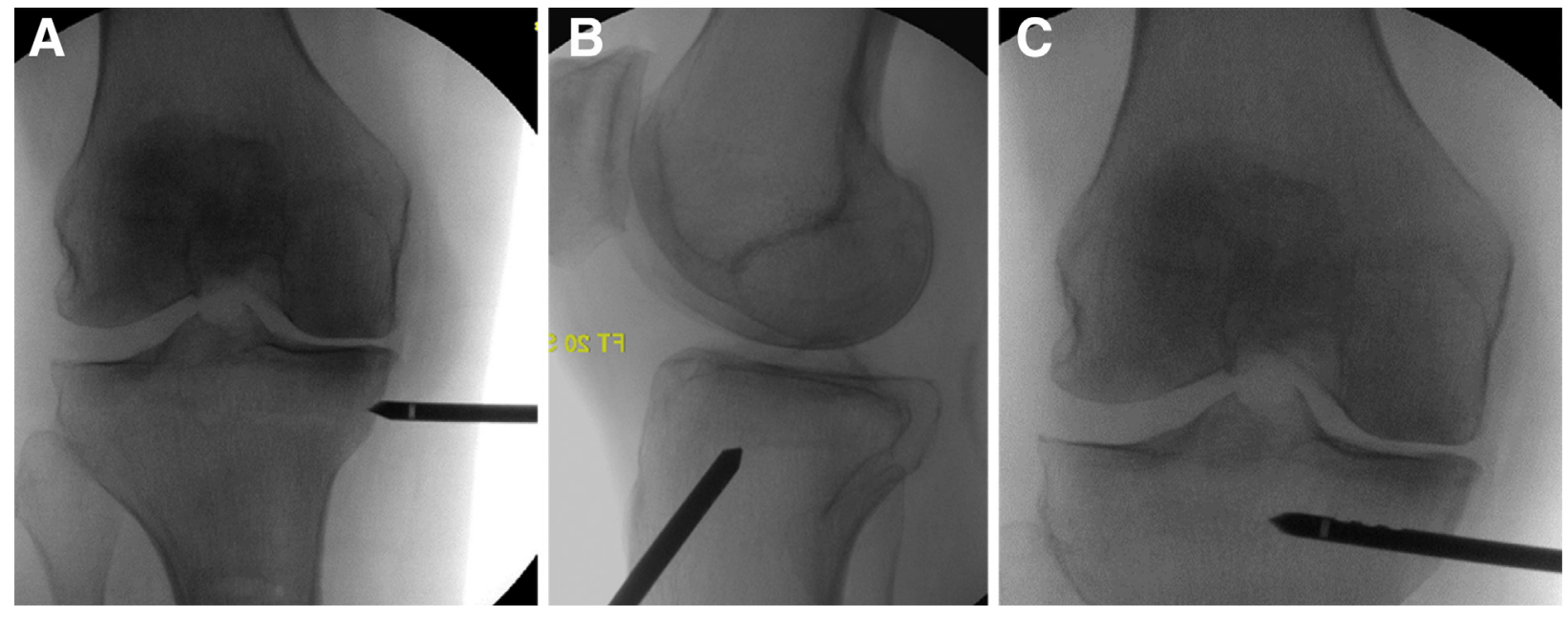

Fig 2. Intraoperative fluoroscopic images of the right knee demonstrating appropriate drill positioning in the anteroposterior (A) and lateral (B) planes, along with drill entry (C) into a bone marrow lesion mapped using magnetic resonance imaging.

\section{Statistical Analysis}

Means and standard deviations were calculated for continuous variables; categorical variables were expressed as a number and percentage. Functional scores (i.e., VAS, IKDC, SF-12) were compared with preoperative values using the Mann-Whitney $U$ test. Statistical significance was set at $P \leq .05$. Kaplan-Meier curves were used for survival analyses. All data were tabulated in Microsoft Office 365-Excel (Version 1710; Microsoft, Redmond, WA), and analyses was conducted using PRISM (version 7; GraphPad, San Diego, CA).

\section{Results}

\section{Patient Cohort}

Twenty patients met the study criteria. No patients declined to participate in the study. Two patients were excluded due to incomplete PROMs. Baseline patient characteristics and demographic data are summarized in Table 1. The age of patients ranged from 38 to 62 years, with a mean of $51.7 \pm 7.9$ years. Fourteen $(70 \%)$ patients had their right knees operated on, and 6 $(30 \%)$ had their left knees operated on. The average preoperative duration of symptoms was $11.7 \pm$ 10.3 months. The mean follow-up was $14.5 \pm 5.5$ (range 6 to 25$)$ months. Seven patients $(35 \%)$ had at least 1 prior surgical procedure on the affected knee. Prior surgical treatment included partial medial meniscectomy $(20 \%)$, partial lateral meniscectomy $(10 \%)$, open meniscectomy $(5 \%)$, meniscal repair $(5 \%)$, microfracture $(5 \%)$, and lateral release $(5 \%)$. Eighteen patients presented with a preoperative diagnosis of SIFK $(90 \%)$, and the other 2 patients $(10 \%)$ presented with SONK (Table 2). Defect location included medial femoral condyle $(65 \%)$, medial tibial plateau $(55 \%)$, lateral tibial plateau $(10 \%)$, and lateral femoral condyle $(5 \%)$. Seven patients $(35 \%)$ had bipolar lesions, resulting in multiple defect locations with a total $>100 \%$. All patients had at least 1 additional concomitant procedure at the time of surgery, as shown in Table 3. Additional intra-articular diagnoses at the time of surgery included medial meniscal tears $(70 \%)$, lateral meniscal tears $(20 \%)$, osteochondral defects $(45 \%)$, loose joint bodies $(15 \%)$, and patellar maltracking $(5 \%)$.

\section{Clinical Outcomes}

The functional outcome of the study group is summarized in Table 4. Preoperative and postoperative functional outcomes scores were available for all 20 patients. There were statistically significant improvements comparing preoperative with postoperative VAS pain scores. IKDC functional outcome scores approached statistical significance.

There were statistically significant improvements when the preoperative VAS pain scores were compared with the postoperative VAS pain scores. Ninety-five percent (19 of 20) of patients experienced improvement in their VAS pain scores from the preoperative baseline. There was an average 5.7-point improvement in pain on a 10-point VAS for pain at 6 months postoperatively. Compared with preoperative values, the VAS decreased from $7.0 \pm 2.1$ to $1.3 \pm 1.5(P=.008)$. This magnitude of improvement in VAS pain scores was clinically meaningful. The subjects met the VAS minimally clinically important difference (MCID) $95 \%$ of the time. For VAS scores, an improvement of 2 points (20 $\mathrm{mm}$ on the $100-\mathrm{mm}$ scale) is considered clinically important (MCIDs). ${ }^{20}$

Table 1. Demographic Data ( $\mathrm{N}=20$ Patients)

\begin{tabular}{lc}
\hline \multicolumn{1}{c}{ Patient Characteristic } & Mean \pm SD or $\mathrm{n}(\%)$ \\
\hline Age, yrs, mean \pm SD (range) & $51.7 \pm 7.9(39-62)$ \\
Sex, male/female, $\mathrm{n}$ & $9(45) / 11(55)$ \\
Body mass index (range) & $28.8 \pm 6.3(18.2-41.9)$ \\
Workers' Compensation & $7(35)$ \\
\hline
\end{tabular}


Table 2. Surgical Data $(\mathrm{N}=20$ Patients $)$

\begin{tabular}{lr}
\hline & $\mathrm{n}(\%)$ \\
\hline BML, type & $18(90)$ \\
SIFK & $2(10)$ \\
SONK & \\
Defect location & $13(65)$ \\
Medial femoral condyle & $1(5)$ \\
Lateral femoral condyle & $11(55)$ \\
Medial tibial plateau & $2(10)$ \\
Lateral tibial plateau & $7(35)$ \\
Patients with bipolar lesions & \\
\hline NOTE. Patients had multiple defect locations (i.e., total $>100 \%)$. \\
BML, bone marrow lesion; SIFK, subchondral insufficiency fracture \\
of the knee; SONK, spontaneous osteonecrosis of the knee.
\end{tabular}

Improvements in IKDC knee functional outcome scores approached statistical significance at final follow-up. Nineteen patients experienced functional improvement of their knee at final follow-up, whereas 1 patient remain unchanged. Postoperative IKDC knee scores increased by an average of 36.9 points $(29.2 \pm 18.9$ preoperatively to $66.1 \pm 20.9$ postoperatively; $P=.063$ ). The resultant magnitude of improvement in IKDC scores was also clinically meaningful. The subjects met the IKDC MCID $70 \%$ of the time. For IKDC scores, an improvement of 11.5 points is considered clinically important. ${ }^{21}$

The overall general health SF-12 scores revealed an improvement from baseline preoperative scores; however, this did not approach statistically significance. Seventeen patients $(85 \%)$ experienced improvement in both the physical and mental component scores. Compared with preoperative values, the Physical Component Score increased from $34.9 \pm 10.2$ to $44.3 \pm$ $12.0(P=.438)$. Likewise, the Mental Component Score showed improvement preoperatively from $51.5 \pm 12.4$ to $55.4 \pm 10.0$ postoperatively $(P=.563)$. The magnitude of improvement for both scores did not reach clinical significance.

Fourteen of the 20 patients $(70 \%)$ had a duration of follow-up of $>1$ year to allow a determination of whether and when patients elected to undergo TKA. Six patients were not included in the analysis due to follow-up duration of $<1$ year. Kaplan-Meier analysis demonstrated a 1-year joint preservation survivorship

Table 3. Concomitant Surgical Procedures ( $N=20$ Patients)

\begin{tabular}{lr}
\hline \multicolumn{1}{c}{ Procedure } & $\mathrm{n}(\%)$ \\
\hline Partial medial meniscectomy & $14(70)$ \\
Chondroplasty & $5(25)$ \\
Partial lateral meniscectomy & $4(20)$ \\
Abrasion arthroplasty & $3(15)$ \\
Loose joint body removal & $3(15)$ \\
Microfracture & $1(5)$ \\
Lateral release & $1(5)$ \\
None & $0(0)$ \\
\hline NOTE. Patients had multiple concomitant procedures (i.e., total \\
$>100 \%)$.
\end{tabular}

Table 4. Preoperative and Final Follow-Up Clinical Outcomes

\begin{tabular}{lcccc}
\hline $\begin{array}{l}\text { Outcome } \\
\text { Measure }\end{array}$ & Preoperative & Postoperative & Change & $P$ Value \\
\hline VAS & $7.0 \pm 2.1$ & $1.3 \pm 1.5$ & $5.7 \pm 0.6$ & .008 \\
IKDC & $29.2 \pm 18.9$ & $66.1 \pm 20.9$ & $36.9 \pm 2.0$ & .063 \\
SF-12 & & & & \\
PCS & $34.9 \pm 10.2$ & $44.3 \pm 12.0$ & $9.4 \pm 1.8$ & .438 \\
MCS & $51.5 \pm 12.4$ & $55.4 \pm 10.0$ & $3.9 \pm 2.4$ & .563
\end{tabular}

NOTE. Data are reported as mean \pm SD; follow-up data include $P$ values for comparisons to preoperative measure using MannWhitney $U$ test.

IKDC, International Knee Documentation Committee; MCS, Mental Component Score; PCS, Physical Component Score; SF-12, 12-Item Short Form Health Survey; VAS, visual analog scale.

of $93 \%$. One patient underwent conversion to TKA at 7.5 months postoperatively.

\section{Radiographic Outcomes}

Based on postoperative MRI, $75 \%$ ( 3 of 4 ) of the BMLs demonstrated complete healing. The 1 patient who did not heal had evidence of moderate worsening of BME of the medial tibial plateau at 6 months postoperatively. In the 3 patients who demonstrated interval healing of their respective BMLs of the knee, all had single lesions. The patients who were able to demonstrate successful healing did not share a common defect location. These BMLs were located in the medial tibial plateau, lateral tibial plateau, and medial femoral condyle.

\section{Discussion}

The main finding of this study was that patients who underwent surgical treatment for BMLs of the knee using BMAC and iDBM showed an improvement in pain and function that persisted in the subsequent 1 year. Overall, there were clinically significant improvements in both VAS pain and IKDC functional outcome scores over the short term. Survival analysis at 1 year showed a $7 \%$ conversion rate to TKA. Whereas the healing rate was $75 \%$ at 6 months, the overall findings provide promising results for this alternative joint preservation surgical procedure in patients with degenerative conditions of the knee.

It is important to consider the biologic options for BMLs of the knee in the context of alternative treatment options. TKA has been shown to lead to reliable pain relief, improved function, and enhanced quality of life. ${ }^{22-24}$ However, it is a major surgical intervention and can be associated with significant complications, increased healthcare costs, and a prolonged postoperative rehabilitation. ${ }^{25-27}$ In the effort for joint preservation, a variety of procedures have been proposed to improve the structural integrity of subchondral bone by stimulating bone repair and remodeling. Subchondroplasty (SCP) was recently investigated for the treatment of subchondral bone defects of the knee. ${ }^{2,3,28-30}$ The SCP technique uses the synthetic 

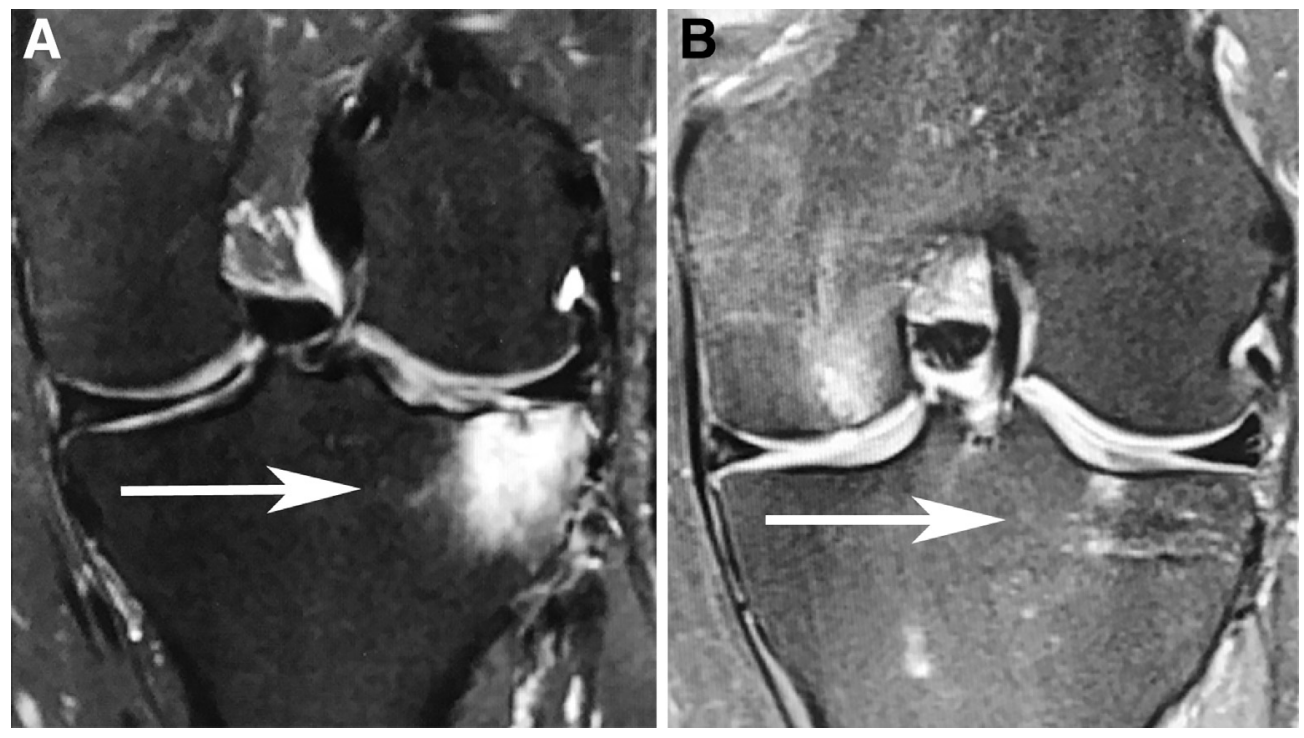

Fig 3. Right knee T2 (A) magnetic resonance image of a 36-year-old man presenting with a subchondral insufficiency fracture of the medial tibial plateau. (B) Magnetic resonance imaging performed 6 months following the procedure demonstrating interval healing of the subchondral insufficiency fracture of the knee.

bone substitute calcium phosphate to target and fill BMLs of the knee. Previous reports in the literature have demonstrated the feasibility of the technique to reduce pain and improve function, with a small risk of complications. $^{2}$ Sharkey et al. ${ }^{31}$ reported pain improvement and a delayed arthroplasty, with resumption of normal activities as soon as 1 week postoperatively. However, the early findings and preliminary results of additional studies reporting outcomes of SCP have been mixed. ${ }^{2,3,28,29,31}$ In addition, filling the void with calcium phosphate bone cement has not been shown to promote physiologic bone remodeling and repair in conjunction with natural healing. ${ }^{32,33}$ This concept may be represented in an initial case series by Chatterjee et al., ${ }^{29}$ which reported a $55 \%$ success rate at a median of 12 months postoperatively in 22 patients treated with SCP. These varied results, along with the need to encourage physiologic bone remodeling to restore normal bony anatomy and function, have led to further options in joint preservation treatment innovation.

Understanding the biology is necessary to understand the treatment. BME has been shown to lead to higher intraosseous pressures, resulting in a reduction of perfusion to subchondral bone. ${ }^{34}$ This decrease is blood flow has been shown to contribute to focal and segmental bone necrosis and cartilage breakdown. ${ }^{35}$ Intraosseous pressures measured in the medial and lateral femoral condyles of patients with BMLs were $97 \%$ greater than pressures in patients without BMLs (63 vs $32 \mathrm{~mm} \mathrm{Hg}$ ). ${ }^{36}$ Prior randomized controlled trials of the hip have shown promising results in patients using BMAC as augmentation for core decompression in the treatment of subchondral bone pathology of the femoral head. ${ }^{18,19} \mathrm{Ma}$ et al. ${ }^{19}$ demonstrated that core decompression augmented with BMAC improved bone healing rates when compared to core decompression alone. Hernigou and Beaujean ${ }^{18}$ demonstrated that only 34 of 189 hips treated for early osteonecrosis progressed to collapse and subsequent total hip replacement at a mean of 7 years after core decompression with bone marrow grafting. This may be because of the osteoinductive, osteoconductive, and osteogenic properties provided by BMAC. BMAC acts as a structural framework for bone growth, and it contains mesenchymal stem cells and growth factors that stimulate bone healing. Such properties may contribute to the balance of bone mineralization that was lost in areas of BMLs of the knee and may modify the course of the disease through physiologic repair (Fig 3). Therefore, applying the principles that we have learned previously in the hip, treatment of BMLs of the knee using BMAC and iDBM appears to be a reasonable alternative for joint preservation in this difficult-to-treat patient population.

Clinically significant changes in PROMs in sports medicine have become increasingly important. The magnitude of improvement in both VAS pain and IKDC functional outcome scores in our study were clinically significant. However, this clinical success may be negatively skewed owing to the high rates of coexisting osteochondral and meniscal disorders that were present in our patient population. All patients had at least 1 additional concomitant intra-articular procedure performed at the time of surgery. The relative contributions for the concomitant procedures were not empirically separated. Therefore, we were unable to definitively conclude whether the aforementioned procedure using BMAC and iDBM is efficacious. However, several studies of subjects with degenerative knee pathology and symptoms similar to the present patient 
population indicate that arthroscopic debridement alone provides no durable relief of osteoarthritic symptoms. ${ }^{37-39}$ The lack of durable pain relief in these studies may have been due to the presence of BMLs, because BMLs are commonly present in this patient population and are not treated with traditional arthroscopy.

\section{Limitations}

There are a few notable limitations to this study that warrant discussion. One limitation is the absence of control cohorts. Because subchondral injection of BMAC and IDBM was always performed along with arthroscopy, the relative contributions of these 2 procedures were not empirically separated. Concomitantly performed arthroscopic procedures likely contributed to some of the observed improvements. A second limitation is the small number of patients and short length of follow-up. Third, there was no prior power analysis to appropriately determine the statistical significance in some PROMs. Last, there was a lack of standard postoperative imaging to assess changes in the BMLs.

\section{Conclusion}

Biologic treatment of BME of the knee using BMAC and iDBM is an effective adjunct to arthroscopy providing short-term pain relief for BMLs associated with degenerative conditions of the knee. This procedure is associated with clinically significant improvements in knee pain and function over a shortterm follow-up.

\section{References}

1. Eriksen EF, Ringe JD. Bone marrow lesions: A universal bone response to injury? Rheumatol Int 2012;32:575-584.

2. Cohen S, Sharkey P. Subchondroplasty for treating bone marrow lesions. J Knee Surg 2015;29:555-563.

3. Farr J, Cohen SB. Expanding applications of the subchondroplasty procedure for the treatment of bone marrow lesions observed on magnetic resonance imaging. Oper Tech Sports Med 2013;21:138-143.

4. Kazakia GJ, Kuo D, Schooler J, et al. Bone and cartilage demonstrate changes localized to bone marrow edemalike lesions within osteoarthritic knees. Osteoarthritis Cartilage 2013:21:94-101.

5. Taljanovic MS, Graham AR, Benjamin JB, et al. Bone marrow edema pattern in advanced hip osteoarthritis: Quantitative assessment with magnetic resonance imaging and correlation with clinical examination, radiographic findings, and histopathology. Skeletal Radiol 2008;37:423-431.

6. Roemer FW, Guermazi A, Javaid MK, et al. Change in MRI-detected subchondral bone marrow lesions is associated with cartilage loss: The MOST Study. A longitudinal multicentre study of knee osteoarthritis. Ann Rheum Dis 2009;68:1461-1465.
7. Madry H, van Dijk CN, Mueller-Gerbl M. The basic science of the subchondral bone. Knee Surg Sports Traumatol Arthrosc 2010;18:419-433.

8. Orth $\mathrm{P}$, Cucchiarini M, Wagenpfeil S, Menger MD, Madry H. PTH [1-34]-induced alterations of the subchondral bone provoke early osteoarthritis. Osteoarthritis Cartilage 2014;22:813-821.

9. Tanamas SK, Wluka AE, Pelletier J-P, et al. Bone marrow lesions in people with knee osteoarthritis predict progression of disease and joint replacement: A longitudinal study. Rheumatology 2010;49:2413-2419.

10. Roemer FW, Neogi T, Nevitt MC, et al. Subchondral bone marrow lesions are highly associated with, and predict subchondral bone attrition longitudinally: The MOST study. Osteoarthritis Cartilage 2010;18:47-53.

11. Wluka AE, Wang Y, Davies-Tuck M, English DR, Giles GG, Cicuttini FM. Bone marrow lesions predict progression of cartilage defects and loss of cartilage volume in healthy middle-aged adults without knee pain over 2 yrs. Rheumatol Oxf Engl 2008;47:1392-1396.

12. Karim AR, Cherian JJ, Jauregui JJ, Pierce T, Mont MA. Osteonecrosis of the knee: Review. Ann Transl Med 2015;3:6.

13. Hussain ZB, Chahla J, Mandelbaum BR, Gomoll AH, LaPrade RF. The role of meniscal tears in spontaneous osteonecrosis of the knee: A systematic review of suspected etiology and a call to revisit nomenclature. Am J Sports Med 2017:036354651774373.

14. Scher C, Craig J, Nelson F. Bone marrow edema in the knee in osteoarthrosis and association with total knee arthroplasty within a three-year follow-up. Skeletal Radiol 2008;37:609-617.

15. Felson DT, Chaisson CE, Hill CL, et al. The association of bone marrow lesions with pain in knee osteoarthritis. Ann Intern Med 2001;134:541-549.

16. Sowers MF, Hayes C, Jamadar D, et al. Magnetic resonancedetected subchondral bone marrow and cartilage defect characteristics associated with pain and X-ray-defined knee osteoarthritis. Osteoarthritis Cartilage 2003;1 1:387-393.

17. Kraus VB, Feng S, Wang S, et al. Subchondral Bone trabecular integrity predicts and changes concurrently with radiographic and magnetic resonance imagingdetermined knee osteoarthritis progression: Bone trabecular integrity predicts OA progression. Arthritis Rheum 2013;65:1812-1821.

18. Hernigou P, Beaujean F. Treatment of osteonecrosis with autologous bone marrow grafting. Clin Orthop 2002;405: 14-23.

19. Ma Y, Wang T, Liao J, et al. Efficacy of autologous bone marrow buffy coat grafting combined with core decompression in patients with avascular necrosis of femoral head: A prospective, double-blinded, randomized, controlled study. Stem Cell Res Ther 2014;5:115.

20. Tubach F. Evaluation of clinically relevant changes in patient reported outcomes in knee and hip osteoarthritis: The minimal clinically important improvement. Ann Rheum Dis 2005;64:29-33.

21. Irrgang JJ, Anderson AF, Boland AL, et al. Development and validation of the International Knee Documentation Committee Subjective Knee Form. Am J Sports Med 2001;29:600-613. 
22. Ethgen $\mathrm{O}$, Bruyère $\mathrm{O}$, Richy F, Dardennes C, Reginster JY. Health-related quality of life in total hip and total knee arthroplasty: A qualitative and systematic review of the literature. J Bone Joint Surg Am 2004;86:963-974.

23. Kane RL, Saleh KJ, Wilt TJ, Bershadsky B. The functional outcomes of total knee arthroplasty. J Bone Join Surg Am 2005;87:1719-1724.

24. Harding P, Holland AE, Delany C, Hinman RS. Do activity levels increase after total hip and knee arthroplasty? Clin Orthop Relat Res 2014;472:1502-1511.

25. Healy WL, Della Valle CJ, Iorio R, et al. Complications of total knee arthroplasty: Standardized list and definitions of The Knee Society. Clin Orthop Relat Res 2013;471:215-220.

26. Iorio R, Robb WJ, Healy WL, et al. Orthopaedic surgeon workforce and volume assessment for total hip and knee replacement in the United States: Preparing for an epidemic. J Bone Joint Surg Am 2008;90:1598-1605.

27. Kurtz S. Projections of primary and revision hip and knee arthroplasty in the United States from 2005 to 2030. J Bone Joint Surg Am 2007;89:780.

28. Bonadio MB, Giglio PN, Helito CP, Pécora JR, Camanho GL, Demange MK. Subchondroplasty for treating bone marrow lesions in the knee: Initial experience. Rev Bras Ortop Engl Ed 2017;52:325-330.

29. Chatterjee D, McGee A, Strauss E, Youm T, Jazrawi L. Subchondral calcium phosphate is ineffective for bone marrow edema lesions in adults with advanced osteoarthritis. Clin Orthop Relat Res 2015;473:2334-2342.

30. Colon DA, Yoon BJV, Russell TA, Cammisa FP, Abjornson C. Assessment of the injection behavior of commercially available bone BSMs for Subchondroplasty ${ }^{\circledR}$ procedures. The Knee 2015;22:597-603.

31. Sharkey PF, Cohen SB, Leinberry CF, Parvizi J. Subchondral bone marrow lesions associated with knee osteoarthritis. Am J Orthop 2012;41:413-417.
32. Winge MI, Reikerås $\mathrm{O}$, Røkkum M. Calcium phosphate bone cement: A possible alternative to autologous bone graft. A radiological and biomechanical comparison in rat tibial bone. Arch Orthop Trauma Surg 2011;131: 1035-1041.

33. Jensen SS, Bornstein MM, Dard M, Bosshardt DD, Buser D. Comparative study of biphasic calcium phosphates with different HA/TCP ratios in mandibular bone defects. A long-term histomorphometric study in minipigs. J Biomed Mater Res B Appl Biomater 2008;90: $171-181$.

34. Kiaer T, Dahl B, Lausten GS. The relationship between inert gas wash-out and radioactive tracer microspheres in measurement of bone blood flow: Effect of decreased arterial supply and venous congestion on bone blood flow in an animal model: Inert gas wash-out and bone blood flow. J Orthop Res 1993;1 1:28-35.

35. Aaron RK, Dyke JP, Ciombor DM, et al. Perfusion abnormalities in subchondral bone associated with marrow edema, osteoarthritis, and avascular necrosis. Ann NY Acad Sci 2007;1117:124-137.

36. Uchio Y, Ochi M, Adachi N, Nishikori T, Kawasaki K. Intraosseous hypertension and venous congestion in osteonecrosis of the knee. Clin Orthop 2001;384: 217-223.

37. Kirkley A, Birmingham TB, Litchfield RB, et al. A randomized trial of arthroscopic surgery for osteoarthritis of the knee. N Engl J Med 2008;359:1097-1107.

38. Moseley JB, O'Malley K, Petersen NJ, et al. A controlled trial of arthroscopic surgery for osteoarthritis of the knee. N Engl J Med 2002;347:81-88.

39. Thorlund JB, Juhl CB, Roos EM, Lohmander LS. Arthroscopic surgery for degenerative knee: Systematic review and meta-analysis of benefits and harms. BMJ 2015;350:h2747. 